\title{
www.tellmed.ch - Das Portal für Ärztinnen und Ärzte
}

\author{
D. Desalmand
}

Das Portal www.tellmed.ch mit freiem Zugang hat das primäre Ziel, Ärztinnen und Ärzten aus Praxis und Klinik mit Hilfe des Internets up to date zu halten und ihnen den Alltag zu erleichtern. Das Schwergewicht liegt dabei auf einem Fachliteratur-Screening, der kontinuierlichen Fortbildung in verschiedenster Form sowie einem vereinfachten Zugang zur weltweiten Literatur aus der Medline-Datenbank. Daneben bietet das Portal eine Palette an medizinischen und gesundheitspolitischen News sowie an elektronisch verfügbaren Hilfsmitteln für den Alltag. Links auf wichtige medizinische Quellen und eine von Exponenten im Gesundheitswesen geschriebene Kolumne runden das Angebot ab.

Korrespondenz:

Dr. med. Daniel Desalmand

Mediscope AG

Eigerstrasse 80

Postfach

CH-3000 Bern 23

Tel. 0313702222

E-Mail: desalmand@mediscope.ch

Internet: www.mediscope.ch
Mit www.tellmed.ch hat die Mediscope AG im April dieses Jahres ein medizinisches Portal lanciert, welches sich an Ärztinnen und Ärzte aus Praxis und Klinik richtet. Das Ziel ist es, dem Arzt als User unter einer URL eine breite Palette an elektronischen Daten für seine tägliche Arbeit und seine individuelle Fortbildung anzubieten. Das Portal wird von Medizinern mit mehrjähriger klinischer Erfahrung, Pharmazeuten und Biologen sowie externen Fachexperten bewirtschaftet. Das Redaktionsteam von Mediscope verfügt über jahrelange Erfahrung in der Aufbereitung von medizinischen und wissenschaftlichen Inhalten für Printprodukte und für das Internet. Der Zugang zu allen Informationen ist

\section{Was bietet Ihnen das Portal konkret?}

Das Portal besteht aus www.tellmed.ch und dem vereinfachten Zugang zu MEDLINE unter www.medline.ch.

\section{www.tellmed.ch}

\section{Fachliteratur}

Ein wesentlicher Teil der redaktionellen Ressourcen fliesst ins Screening der bekannten medizinischen Fachzeitschriften (New England Journal of Medicine, Lancet, British Medical Journal, Journal of the American Medical Association, Archives und Annals of Internal Medicine, um nur die renommiertesten zu nennen). Innert Stunden oder wenigen Tagen nach Erscheinen der aktuellen Ausgabe finden sich unter Journalscreening deutsche Zusammenfaskostenlos. sungen der klinisch relevanten Artikel, sofern diese gewisse EBM-Kriterien erfüllen. Selbstverständlich sind die Quellen und Links zu Artikeln zum Thema angegeben, sobald diese in der Medline verfügbar sind. Das Ziel des Redaktionsteams ist die Erstellung einer guten Übersicht über die wichtigen medizinischen Publikationen und verfolgt nicht bloss die Wiedergabe von Leckerbissen.

Unter Studienbesprechungen werden wichtige Studien, mit einer etwas grösseren Zeitlatenz, im Sinne eines «critical appraisal» eingehend besprochen und beurteilt, und zwar vom Redaktionsteam oder von einem externen Fachexperten.

Ausserdem hat der Interessierte unter diesem Hauptnavigationspunkt unter Medizin Spektrum das Online-Archiv aller bisher erschienenen Ausgaben der gleichnamigen Zeitschrift der Mediscope AG. Diese entsteht alle 2 Monate in Zusammenarbeit mit einer Schweizer Klinik und behandelt wichtige medizinisch aktuelle Fragestellungen zu einem ausgewählten Fachgebiet. In Form von PDF-Dokumenten findet sich hier auch eine Auswahl an Fachzeitschriften aus dem Rosenfluh Verlag, so zum Beispiel ArsMedici, Pädiatrie, Managed Care und andere.

\section{Fortbildung}

Jedermann spricht von E-Learning. E-Learning heisst Bildung, Weiter- oder Fortbildung unter Zuhilfenahme der neuen Medien. Diese Art der Vermittlung von Lerninhalten wird bereits seit vielen Jahren praktiziert. Abgesehen vom erwähnten Journalscreening und den Studienbesprechungen bietet Mediscope unter www. tellmed.ch verschiedene diagnostische Quiz (EKG, Röntgen, Labor) und Zusammenfassungen von SGIM- und SGAM-anerkannten medizinischen Tagungen in der Schweiz an, immer mit dem Ziel, in möglichst kurzer Zeit einen maximalen Lerneffekt zu erzielen.

Geplant sind auch isolierte Online-Fortbildungskurse, deren Absolvierung durch ein Zertifikat bestätigt werden kann. Der Vorteil der reinen Online-Angebote beschränkt sich nicht nur auf die vielzitierte zeitliche und örtliche Unabhängigkeit. Sobald das Angebot an Fortbil- 
dungskursen auf dem Netz eine gewisse Vielfalt erreicht hat, wird sich jeder Interessent seine für sich optimale Fortbildung zusammenstellen können. Das Angebot kann vor Absolvierung eines ganzen Kurses kurz geprüft werden, und das Risiko, Stunden in einer unattraktiven Fortbildung zu «sitzen», besteht nicht. Zudem kann der Lernerfolg durch einen Online-Test ermittelt werden, als Feedback für den User.

\section{Kongresse}

Das Redaktionsteam der Mediscope AG besucht jedes Jahr eine ganze Anzahl nationaler und vor allem internationaler Kongresse. Kurzberichte und zum Teil tiefergehende Zusammenfassungen einzelner Referate sollen die neuesten Erkenntnisse in einem Fachgebiet an den Leser weitervermitteln.

\section{Tools}

Diese Rubrik ersetzt die Schublade mit den verschiedenen Berechnungstabellen, diagnostischen Scores und Formularen. Von der OnlineBerechnung der Kreatininclearance oder des korrigierten Serum-Kalziums über diagnostische Scores zur Abschätzung der Wahrscheinlichkeit einer Venenthrombose bis zu Impfempfehlungen, BAG-Formularen und Hilfen beim Patientenmanagement (Asthmatagebuch, Kopfwehkalender) findet sich dort alles für den Praxisalltag. Auch Hinweise zu Software für den Taschencomputer fehlen nicht.

\section{Presse und Kolumne}

Hier finden sich medizinische News aus der Laienpresse und Aktuelles aus der Gesundheitspolitik, möglichst auf den Arzt fokussiert.

Besonders empfohlen sei ausserdem die Lektüre der Kolumne unter der Federführung von Prof. K. Neftel, wo sich Exponenten im Gesundheitswesen, aus ganz verschiedenen Richtungen, die Ehre geben.

\section{Links}

Einer der grossen Vorteile des Internets ist die Nähe zu anderen Informationsquellen. Die Links sind eingeteilt in vier verschiedene Kategorien: Datenbanken und Quellen (Arzneimittelkompendium, Arzneimittelinteraktionen, Dermatologieatlas, Toxi, PubMed usw.), Schweizerische Fachgesellschaften, Literatur und Organisationen/Institutionen.

\section{Newsletter}

Eine Zusammenstellung aller wichtigen, aktuellen Inhalte soll in Zukunft via Newsletter regel- mässig an alle Interessierten kostenlos versandt werden. Der Eintragungsort für den Newsletter findet sich auf der Einstiegsseite von www. tellmed.ch. Die E-Mail-Adressen werden ausdrücklich nicht an Dritte weitergegeben.

\section{Link zum Konsumentenportal www.sprechzimmer.ch}

Zeitgleich zum Ärzteportal hat Mediscope auch ein Konsumentenportal lanciert: www.sprechzimmer.ch bietet einem Laienpublikum fachlich fundierte, topaktuelle Informationen rund um die Themen Gesundheit und Wohlbefinden, Prävention und Aufklärung sowie Gesundheitspolitik und News. In allgemein verständlicher Sprache werden neuste Behandlungsmethoden erklärt und medizinische Erkenntnisse oder Studien seriös durch das aus Fachleuten bestehende Redaktionsteam aufbereitet. Damit tritt www.sprechzimmer.ch mit kompakter Information gegen die qualitativ stark variierende Publikationsflut rund um heutige Gesundheitsthemen an.

Auch für Fachpersonen finden sich wertvolle Dienstleistungen auf dem Konsumentenportal, die letztendlich wieder den Patienten zugute kommen: Wichtige Adressen, Anlaufstellen und Selbsthilfegruppen sowie Patientenbroschüren können einfach und zeitsparend abgerufen werden. Auf www.tellmed.ch befindet sich ein entsprechender Link zum Konsumentenportal.

\section{medline.ch}

Nicht weniger als 15 Millionen Publikationen sind in der Medline-Datenbank abgelegt, doch wie sollen relevante Publikationen $\mathrm{zu}$ einer klinischen Fragestellung in dieser Menge gefunden werden? Mediscope hilft dem User mit medline.ch, die aufwendige, anspruchsvolle Suche dank einer übersichtlichen Navigation und eines einfachen Suchmechanismus wesentlich zu vereinfachen.

Durch vordefinierte Suchalgorithmen mit einem klinisch praktischen Fokus wird der Suchende schnell und fachspezifisch fündig. Ähnlich wie in der PubMed besteht die Möglichkeit, Filter für zum Beispiel lediglich diagnostische oder therapeutische Studien einzubauen oder die Suche zeitlich einzugrenzen. Selbstverständlich kann auch mit Schlagwörtern gesucht werden, sei dies über das ganze Portal, in einem Fachgebiet oder spezifisch für ein in der Navigation definiertes Krankheitsbild. Da die Literatur in der Medline englisch hinterlegt ist, müssen auch englische Suchbegriffe eingegeben werden. 


\section{Redaktionelle Unabhängigkeit und Kommerz}

Beide Portale werden durch das Redaktionsteam von Mediscope betreut. Die Auswahl der Artikel erfolgt nach klaren Richtlinien und obliegt einzig und alleine dem Redaktionsteam. An die Redaktion zur Veröffentlichung gesandte Beiträge werden inhaltlich geprüft und bei Verwendung mit einem klaren Verweis auf die Verfasser/Quelle versehen. Neben Bannerwerbungen und Anzeigenschaltungen werden einzelne Unterrubriken gezielt von Unternehmen gesponsert - und dadurch erst ermöglicht. Diese Informationen bzw. Unterrubriken werden transparent gekennzeichnet (mit freundlicher Unterstützung von, im Auftrag von); das Redaktionsteam oder extern zugezogene Experten zeichnen für die neutrale, unabhängige und ausgewogene Informationszusammenstellung verantwortlich.

\section{Blick in die Zukunft}

Dort, wo früher ein Informationsportal nach dem anderen aus dem Boden schoss, herrscht heute gähnende Leere. Man hat vor allem das finanzielle Potential solcher Projekte stark über- schätzt. Allerdings wird kaum jemand bestreiten, dass es sich beim Internet um ein äusserst wertvolles Medium handelt, das über kurz oder lang Einzug in jeden Berufszweig halten wird, so auch in die ärztliche Tätigkeit. Immer häufiger muss sich auch der Grundversorger gegenüber Patienten, Krankenkassen und nicht zuletzt Juristen bezüglich seines Handelns rechtfertigen. Dies setzt insbesondere ein breites, immer wieder aktualisiertes Fachwissen, aber auch die Kenntnis des medizinisch-gesundheitspolitischen Umfelds voraus. Wir hoffen, den praktizierenden Arzt darin mit unserem Portal unterstützen zu können.

www.tellmed.ch ist noch ein sehr junges Portal, die Entwicklungsmöglichkeiten dafür um so grösser. Insbesondere im Bereich Fortbildung/E-Learning ist noch viel zu tun. Das Angebot ist - zumindest hierzulande - zurzeit noch sehr gering und es müssen zuerst Erfahrungen gesammelt werden. Es ist aber durchaus denkbar, dass in einigen Jahren Online-Fortbildungskonti geführt werden, wo die Absolvierung von Fortbildungsstunden erfasst werden und direkt an die Kontrollstellen geleitet werden kann.

Das Redaktionsteam ist bemüht, das Portal laufend zu erweitern und täglich zu aktualisieren. Wir freuen uns über Wünsche, Anregungen und konstruktive Kritik.

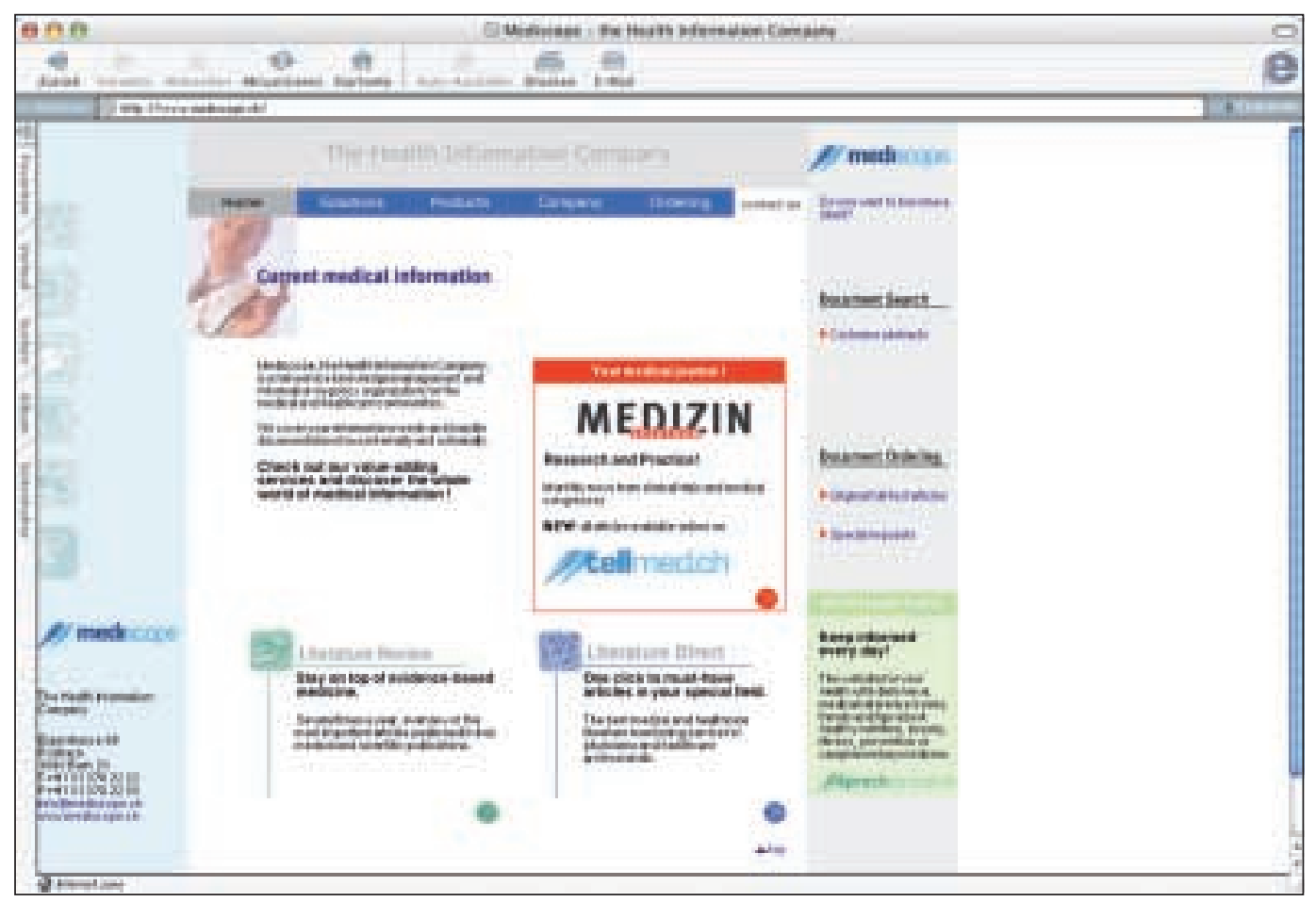

